of the first class, and gastric irritation from irritating matters and worms, and painful menstruation on the other.

For spinal irritation, therefore, a term which should pro. bably be expunged altogether from medical etiology, we may, I think, substitute peripheral irritation of one kind or other-a really intelligible and meaning term-in some if not many of the cases sought to be explained by the former.

Then we have paralysis agitans, in some persons with semiworn.out cerebral power, evidently one phase of the choreal or clonic state of the muscles.

From a careful examination of this subject, I find it difficult to conclude that any more direct relation exists between chorea and carditis than the one now explained; and I do not think it at all necessary to this inquiry, even if it were nossible to attempt anything in the way of explanation as to that mysterious connexion between the cardiac and other ganglia of the sympathetic and the cerebral and true spinal system, nor of the modus operandi of the influence which an inflamed heart would exercise through this relation upon the brain and nerrous system.

\section{CASES ILLUSTRATIVE OF THE INFLUENCE OF BELLADONNA.}

By Richand Hughes, M.R.C.S., L.R.C.P.Ed.(Exam.), Surgeon to the Brighton Orthopedic Hospital.

In tro papers-one contained in the British Medicar. Journar for June 2nd, 1860), and the other in the London Medical Review for August 1860 - I have discussed at some length the physiological influence of belladonna. The conclusions to which I have there come are, that this drug is an excitant of the sympathetic nervous system, and a depressant (mainly by local action) of the cerebro-spinal system.

In the following paper, I propose to exhibit some results of the practical application of this theory, and to point out the farther indications for practice thereby afforded. And I will begin with cases where the action of this drug upon the sympathetic system appears to be the rationale of its curative influence.

CASE I. Enuresis. July 1859. George M., aged 14, had long suffered from nocturnal incontinence of urine. There was nothing particular about the general appearance of the patient, or that of his urine. I ordered small doses of tincture of cantharides, which relieved him temporarily; but immediate relapse followed its discontinuance. I now prescribed onethird of a grain of extract of belladonna twice a day, in cinnamon-water. In three days his complaint left him; a slight relapse, about a week subsequently, was immediately removed by a few more doses of the medicine; and he has since been entirely free of his ailment.

Remarks. Incontinence of urine may obviously result from two immediate causes. 1. From over-action of the longitudinal fibres of the muscular coat of the bladder (detrusor urinæ). This is usually excited by some irritating quality of the urine, and is best removed by alkaline medicines and opiate supposi. tories. 2. From weakness or paralysis of its sphincter. 'This second and far more common cause of enuresis, usually met with in delicate children, requires for its cure some agent capable of toning the sphincter muscle, either immediately, or through the medium of its nerves. Such an agent we have, according to my views, in belladonna; and, accordingly, the accounts of the influence of this drug over the disease from all parts of Great Britain and the Contineut are highly encouraging. It rarely fails to overcome it, and in its use is subject to none of the evils occasionally produced by cantharides.

In prescribing this drug for children, the discovery of Dr. Fuller as to its comparatively slight effects on young subjects must be borne in mind; and what would seem to be large doses must be unhesitatingly used.

CASE II. Milk.Abscess. July 1860. Emily H., aged 24, nine weeks after continement caught cold. When I saw her, I found at the upper part of the left breast a hard swelling elevated above the surface, of about the size of a hen's egg; it was very painful, red, hot, and tender to the touch. 'There was also pain in the right side, constipation, headache, high fever; and, in the course of the preceding night, there had been some delirium. The fever had somewhat abated since vomiting a quantity of pure bile early in the morning. These symptoms pointed to concomitant derangement of the liver, and soon yielded to a mercurial pill and saline draught. The state of the breast 1 treated by suspending the mamma by a handkerchief tied over the shoulder, and keeping the swelling covered with the unguentum belladonnæ of the Pharmacopoia. In twelve hours, all pain had ceased, and there had been no increase of swelling. Next day, the redness and heat had gone, some tenderness only remaining. The swelling remained stationary and slightly tender for a few days, then slowly disappeared. The infant was not put to the breast during the mother's illness, and the general fever was suffi. cient to diminish the secretion of milk; but, as soon as she became convalescent, the flow of milk in both breasts returned as freely as before.

Remarks. The localised inflammation of the breast to which (from its great tendency to suppuration) this term " milkabscess" is commonly applied, has now been frequently treated by belladonna. 'This drug has been ascertained to possess galactifuge properties; and accordingly, being applied in the form of extract or ointment around the nipple in these cases, it speedily checks the secretion of milk, and with it the inflammation (British Medical Journal, Feb. 6, May 29, June 12, 1858 , etc.).

The galactifuge properties of belladonna are easily ex plicable upon the theory of its action which I have advanced. Cl. Bernard has found that there are two ways of diminishing the secreting power of a glard-1, galvanising the sympathetio nerves which supply its blood-vessels; 2, dividing the cerebrospinal nerves which supply its secreting cells. Belladonna, according to my view of it, both excites the sympathetic (as by galvanism), and depresses the cerebro-spinal influence (as by section) of the parts which it affects. The result of its application to a gland like the breast will therefore be to diminish alike its secreting power and the supply of the material on which that power is exercised. And this galactifuge property of the drug will be found invaluable in cases of still-lirth, premature wearing, and others, where the check of secretion of milk is loudly called for.

But it will be noticed that, in the case I have recorded, there was no check of the secretion of milk. Not to speak of the superior practical advantages of a plan of treatment which avoids this unpleasant concomitant, it is obvious that the rationale of the cure must be a different one. It seems to me to resolve itself into the simple action of belladonna in exciting the vaso-motor nerves, and thereby contracting the capillaries. The essence of the inflammatory condition, to my thinking, is a morbidly dilated state of these vessels. The exudation from them, on which Professor Hughes Bennett and his school lay so much stress, is surely a consequence only of their preternatural dilatation and fulness. A substance which will contract them will save them the necessity of relieving themselves by exudation at all, and, even when this has taken place, will most surely promote its absorption. And this was the view of John Hunter. "If," he wrote in 1794, "we could discover an agent which would cause the contraction of the extreme vessels, we should have in this the true remedy for inflammation."

This case, then, of cure of threatened milk-abscess by the local application of belladonna, appears to open a wide field for the treatment of inflammation in general. The three indications which inflammation gives us for treatment are-1. To moderate the force and frequency of the heart's action; 2 . To diminish the superfibrination of the blood; 3 . 'To reduce the hyperæmia of the part affected. The old mode of effecting the first two objects-in other words, of relieving the symptomatic inflammatory fever-was to bleed, and to give mercury; which may fairly be described as robbing a patient of a portion of his life, and poisoning the remainder. A more enlightened practice fulfils the former indication by antimony, aconite, and the other substances which have this physiological effect upon the heart; and the latter by low diet, diluents, and salines.* The third indication - the reduction of the hyperæmia of the partis commonly met by local bleeding and cold. But these are measures only applicable in their fulness to external inflammations, and even there are open to much improvement. Local bleeding, however efficacious, wastes blood, and leaves the capillaries weak and still morbidly dilated; and cold must be continuously applied, to be of avail. We should gain immensely, could we find a remedy capable of acting, whether locally or through the general system, upon the nerves of the inflamed part, so that its dilated capillaries might be aided to that contraction for which Nature is striving, and thus the hyperæmia of the part reduced without the loss of a drop of blood, or the inconveniences inseparable from leeches, cupping, and the continuous application of cold.

* I find nitre, which combines both these actions, an admirable genera remedy for the mild fevers and inflammations of chiluhood. 
Such an agent I believe we have in belladonna; and I augur an immense advance in our control over inflammation as its use therein becomes extended within the limits which practical experience alone can define. (See the Lancet for Aug. 4, 1860, for a case of myelitis treated by belladonna applied along the spine.)

Case III. Congestive Headache. August 1860. Mrs. M., aged $7 t$, for four days in succession had been visited with severe headache, beginning with blindness, and lasting the greater part of the day. Similar headaches, some years ago, had been relieved by leeching. The administration of belladonna was commenced at the first coming on of the blindness, on the fifth day. The attack this day passed off sooner than usual. On the sixth day, there was but a slight attack; and since then there have been none.

liemanks. 'The remarks made upon the last case will in most respects apply here, substituting congestion for inflammation. The congestive character of the headaches seemed marked-1, by the accompanying blindness; 2 , by the relief formerly afforded by leeches; 3 , by the absence of good effect from a full dose of Hotfman's anodyue-a medicine which I find invaluable in cases of simple nervous headache. Here the bellatonna accomplished the same good end as the leeches, without their expense, inconvenience, or spoliation of the vital fluid.

The result of this case would seem to indicate the use of belladonna to remove that habitual tendency to congestion of the brain which usually results in apoplexy.

[To be continued.]

\section{Grogues of allotical Sirme: \\ AT HOME AND ABROAD.}

\section{SURGERY.}

P R O S T A T O R R H A A.

UNDER the title of Practical Observations on the Nature and Treatment of Prostatorrhœa, Dr. S. D. Gross has published, in the North American Medico-Chirurgical Review for July, a paper on an affection which does not appear to have been hitherto distinctly described by surgical writers, although it is of sufficiently frequent occurrence. In reference, however, to the fact of its description, a correspondent of this Jounnal, if wo recollect rightly, described its symptoms a year or two ago; but we cannot, at the present time, give the reference to the contribution. As, then, the essay of Dr. Gross is the first in which we remember to have seen the disorder distinctly described and localised, we shall reprint the greater part of it.

Prostatorrhoi is, as the term implies, a discharge from the prostate gland, generally of a thin mucous character, dependent upon irritation, if not actual inflammation, of the component tissues of that organ. The reason why the disease has not hitherto received any specific name or place in surgical nomenclature, is simply because it has always been confounded with other lesions, as gleet, or chronic urethritis, seminal losses, and cystorrhœa, or chronic inflammation of the mucous membrrne of the bladder; from which, in fact, it is often difficult to distinguish it.

Dr. Gross has not met with prostatorrhœa in children or very young subjects, probably because all kinds of diseases of the prostate are so very rare at that period of life. That it may occur, however, even at a very tender age, is altogether likely, especially in children labouring under stone in the bladder, prolapse of the bowel, or worms in the rectum, causing the organ to suffer from reflected irritation. After the twentieth year the disease is sufficiently common, and instances are occasionally met with even in very old persons. As long as the prostate gland remains small and inactive, or is not brought fully under the influence of the sexual organs, with which it is so intimately associated, it is comparatively infrequent.

Dr. Gross is not able to say, from his experience, what classes of persons are most liable to suffer from this affection; but it has seemed to him that it is most frequent in those of a sanguineo-nervous temperament, with strong sexual propensities, leading to the frequent indulgence of the venereal appetite, if not to positive venereal excesses, either in the natural manner or by masturbation. An irritation would thus seem to be established in the prostate gland, attended with more or less discharge of its peculiar secretion, either in a normal or abnormal state. Single and married men are, apparently, equally prone to it. Once established, it is probable that certain occupations may serve to keep it up; and it is also likely that there are certain employments which may predispose to it, although it would require a much longer experience than what is possessed by any one individual to point them out in a definite or satisfactory manner. Intemperance in eating and drinking, frequent horseback exercise, sexual abuse, and disease of the bladder, anus, aud rectum, may all be regarded as contributing to such a resilt.

The exciting causes of prostatorrhœe are not always very evident. In most of the cases that have fallen under my observation, the affection was traceable, either directly or indirectly, to venereal excesses, chronic inflammation of the neck of the bladder, stricture of the urethra, or disease of some kind or other of this canal. In some cases it has its origin in disorder of the lower bowel, as hemorrhoids, prolapse, fissure, fistulæ, ascarides, or the lodgment of some foreign body. It is easy to conceive how reflected irritation might induce this dis. ease. The connection between the prostate gland and anorectal region is very close and intimate, and hence whatever affects the one will almost be sure, in time, to implicate the other, either in consequence of proximity of structure, or as an effect of the laws of sympathy. However this may be, no judicious surgeon ever omits to examine these parts most thoroughly in the event of any serious disease of any of them before he attempts a course of treatment. Temporary prostatorrhora is occasionally excited by the exhibition of internal remedies, as drastic cathartics, cantharides, and spirits of turpentine; or, in short, whatever has a tendency to invite a preternatural aftlux of blood to the prostate gland and neck of the bladder, or to the posterior portion of the urethra. Another cause of the disease, and, according to Dr. Gross's experience, a very common one, especially in young men, is masturbation or self-pollution.

The symptoms of prostatorrhœa are sufficiently characteristic. The most prominent, as already stated, is a discharge of mucus, generally perfectly clear and transparent, more or less ropy and of varying quantity, from a few drops to a drachm and upwards, in the twenty-four hours. It is seldom that it is puriform, and still more rare that it is purulent. When considerable, the flow keeps up almost a constant moisture at the orifice of the urethra, and may even make a decided impression upon the putient's linen, leaving it wet and stained, somewhat in the same manner as in gleet or gonorrhca, though in a much less marked degree. The most copious evacuations of this kind generally occur while the patient is at the water-closet, engaged in straining, especially if the bowels are constipated, or the facal matter is uncommonly hard, or greatly distends the rectum, so as to exert an unusual amount of pressure upon the prostate gland.

The discharge, whether small or large, is often attended with a peculiar tickling sensation, referred by the patient to the prostate gland, from which it frequently extends along the whole length of the urethra, and even to the head of the penis. In some cases, indeed in many, the feeling is of a lascivious, voluptuous, or pleasurable nature, not unlike that which accompanies the earlier stages of sexual intercourse. Not a few patients experience what they call a "dropping sensation," as if the fluid fell from the prostate gland into the urethra. Other anomalous symptoms often present themselves, such as a feeling of weight and fatigue in the region of the prostate, the anus, and rectum, or along the perineum, with, perhaps, more or less uneasiness in voiding urine, and a frequent desire to empty the bladder; some patients are troubled with morbid erections, and their sleep is interrupted with lascivious dreams.

It is astonishin" how much the patient's mind often suffers in this affection. The discharge, even if ever so insignificant, occasions him the greatest possible disquietude; for at one time he imagines that it is a source of much bodily debility, or that it is productive of weakness and soreness in the dorsolumbar region, especially if these symptoms happen to coexist; at another, that he is about to become impotent, under the delusive idea that the flow is one of a seminal character; an idea which not unfrequently haurits him day and night, and from which hardly anything can, perhaps, even temporarily divert his attention. His mind, in short, is poisoned, and the consequence is that he is incessantly engaged in trying to obtain relief, running from one practitioner to another, distrusting all, 